This is the peer reviewed version of the following article: Deiteren, A., De Man, J. G., Keating, C., Jiang, W., De Schepper, H. U., Pelckmans, P. A., Francque, S. M. and De Winter, B. Y. (2015), Mechanisms contributing to visceral hypersensitivity: focus on splanchnic afferent nerve signaling. Neurogastroenterology \& Motility, 27: 1709-1720, which has been published in final form at doi:10.1111/nmo.12667. This article may be used for non-commercial purposes in accordance with Wiley Terms and Conditions for Self-Archiving. 


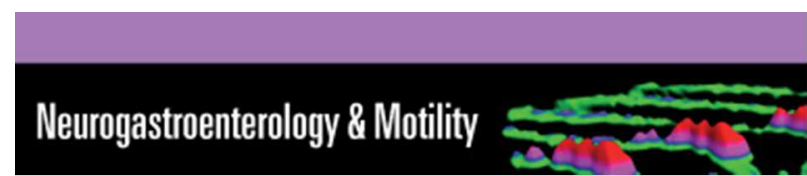

\section{Mechanisms contributing to visceral hypersensitivity: focus on splanchnic afferent nerve signaling}

\begin{tabular}{|c|c|}
\hline Journal: & Neurogastroenterology and Motility \\
\hline Manuscript ID: & NMO-00082-2015.R1 \\
\hline Manuscript Type: & Original Article \\
\hline Date Submitted by the Author: & $11-J u l-2015$ \\
\hline Complete List of Authors: & $\begin{array}{l}\text { Deiteren, Annemie; University of Antwerp, Laboratory of Experimental } \\
\text { Medicine and Pediatrics, Division of Gastroenterology } \\
\text { De Man, Joris; University of Antwerp, Laboratory of Experimental Medicine } \\
\text { and Pediatrics, Division of Gastroenterology } \\
\text { Keating, Christopher; University of Sheffield, Biomedical Science } \\
\text { Jiang, Wen; University of Sheffield, Biomedical Science } \\
\text { De Schepper, Heiko; University Hospital of Antwerp, Gastroenterology \& } \\
\text { Hepatology; University of Antwerp, Laboratory of Experimental Medicine } \\
\text { and Pediatrics, Division of Gastroenterology } \\
\text { Pelckmans, Paul; University of Antwerp, Laboratory of Experimental } \\
\text { Medicine and Pediatrics, Division of Gastroenterology; University Hospital } \\
\text { of Antwerp, Gastroenterology \& Hepatology } \\
\text { Francque, Sven; University Hospital of Antwerp, Gastroenterology \& } \\
\text { Hepatology; University of Antwerp, Laboratory of Experimental Medicine } \\
\text { and Pediatrics, Division of Gastroenterology } \\
\text { De Winter, Benedicte; University of Antwerp, Laboratory of Experimental } \\
\text { Medicine and Pediatrics, Division of Gastroenterology }\end{array}$ \\
\hline Key Words: & $\begin{array}{l}\text { mechanosensitivity, afferent nerve signaling, visceral hypersensitivity, } \\
\text { colitis, splanchnic nerves }\end{array}$ \\
\hline
\end{tabular}


1

2

3

4

5

6

7

8

10

11

12

13

14

15

16

17

18

19

20

21

22

23

24

25

26

27

28

29

30

31

32

33

34

35

36

37

38

39

40

41

42

43

44

45

46

47

48

49

50

51

52

53

54

55

56

57

58

59

60

1. Title page

MECHANISMS CONTRIBUTING TO VISCERAL HYPERSENSITIVITY: FOCUS ON SPLANCHNIC AFFERENT NERVE SIGNALING

Short running page heading: Splanchnic afferent nerve signaling in colitis

Annemie Deiteren ${ }^{1}$, Joris G. De Man ${ }^{1}$, Christopher Keating ${ }^{2}$, Wen Jiang ${ }^{2}$, Heiko U. De Schepper ${ }^{1,3}$, Paul A. Pelckmans ${ }^{1,3}$, Sven M. Francque ${ }^{1,3}$, Benedicte Y. De Winter ${ }^{1}$

${ }^{1}$ Laboratory of Experimental Medicine and Pediatrics, Division of Gastroenterology, University of Antwerp, Antwerp, Belgium; ${ }^{2}$ Department of Biomedical Sciences, University of Sheffield, Sheffield, UK; ${ }^{3}$ Antwerp University Hospital, Department of Gastroenterology and Hepatology, Antwerp, Belgium.

Author for correspondence:

Benedicte De Winter

University of Antwerp, Campus Drie Eiken

Laboratory of Experimental Medicine and Pediatrics, Division of Gastroenterology

Universiteitsplein 1, B-2610 Antwerp, Belgium

Email: benedicte.dewinter@uantwerpen.be

Phone: +32 326527 10; Fax: +32 32562567 
Deiteren et al - 2

\section{Abstract}

Background: Visceral hypersensitivity is a main characteristic of functional bowel disorders and is mediated by both peripheral and central factors. We investigated whether enhanced splanchnic afferent signaling in vitro is associated with visceral hypersensitivity in vivo in an acute and postinflammatory rat model of colitis.

Methods: TNBS-colitis was monitored individually by colonoscopy to confimr colitis and follow convalescence and endoscopic healing in each rat. Experiments were performed in controls, rats with acute colitis and in post-colitis rats. Colonic afferent mechanosensitivity was assessed in vivo by quantifying visceromotor responses (VMRs), and by making extracellular afferent recordings from splanchnic nerve bundles in vitro. Multi-unit afferent activity was classified into single units identified as low threshold (LT), wide dynamic range (WDR), high threshold (HT) and mechanically insensitive afferents (MIA).

Key results: During acute TNBS-colitis, VMRs were significantly increased and splanchnic nerve recordings showed proportionally less MIA and increased WDR and HT afferents. Acute colitis gave rise to an enhanced spontaneous activity of both LT and MIA and augmented afferent mechanosensitivity in LT, WDR and HT afferents. Post-colitis, VMRs remained significantly increased while splanchnic nerve recordings showed that the proportion of LT, WDR, HT and MIA had normalized to control values. However LT and MIA continued to show increased spontaneous activity and WDR and HT remained sensitized to colorectal distension.

Conclusions \& Inferences: Visceral hypersensitivity in vivo is associated with sensitized splanchnic afferent responses both during acute colitis and in the post-inflammatory phase. However splanchnic afferent subpopulations are affected differentially at both time points.

Keywords: mechanosensitivity, afferent nerve signaling, visceral hypersensitivity, TNBS, colitis, splanchnic nerves 


\section{Key messages:}

- The role of splanchnic afferent nerves in visceral hypersensitivity remains unclear.

- Visceral sensitivity during acute TNBS-colitis and in the post-inflammatory phase was assessed in vitro by recording splanchnic afferent nerve discharge and in vivo by recording visceromotor responses to colorectal distension.

- Sensitization of splanchnic afferents in vitro is associated with visceral hypersensitivity in vivo both during acute colitis and in the post-inflammatory phase but the different subpopulations of afferent nerves are differentially affected in the acute versus the postinflammatory phase of colitis. 
Deiteren et al - 4

\section{Introduction}

Visceral hypersensitivity is a main characteristic of bowel disorders such as inflammatory bowel disease (IBD) or irritable bowel syndrome (IBS) (1-4). It is involved in pain perception and is mediated by both peripheral and central factors. Growing evidence suggests that sensory nerve pathways play a key role in the peripheral pain transmission.

Sensory information from colon and rectum is conveyed via thoracolumbar splanchnic nerves and lumbosacral pelvic nerves to the spinal cord that synapse onto second-order neurons in the spinal cord, which in turn transmit the sensory signal to the higher brain regions (5). Various studies have shown that lumbosacral pelvic nerves are the main signaling pathway for visceral sensory processing under physiological conditions including peristaltic movement of fecal contents into the rectum; in contrast, thoracolumbar splanchnic nerves convey primarily noxious signals derived from rapid gut distension and mesentery torsion (6-11). This pathway is less active under physiological conditions.

Using different animal models, several studies corroborate the involvement of both pelvic and splanchnic afferents in mediating visceral hypersensitivity, however splanchnic afferents seem specifically equipped for noxious nerve signaling during inflammation $(6,9,12-16)$. For instance, splanchnic afferents are more likely to respond to inflammatory mediators such as adenosine $5^{\prime}$ triphospate (ATP) and bradykinin and more frequently increase their firing rate when exposed to capsaicin $(8,17)$. Moreover, silent nociceptors recruited by these mediators were exclusive to the splanchnic signaling pathway $(8,17)$. In addition, electrophysiological recordings in mice corroborated the predominant involvement of splanchnic afferents in acute trinitrobenzene sulphonic acid (TNBS)-colitis (6). In contrast, splanchnic afferent discharge in rats remained unaffected by dextran sulphate sodium (DSS)-colitis $(18,19)$. In addition, Chen et al. (20) recently demonstrated that sensitized responses of spinal afferents do not always result in visceral hypersensitivity in vivo as assessed using behavioral assays such as the visceromotor response (VMR) to colorectal distension. Besides, acute colitis can induce long-lasting visceral hypersensitivity which persists after the initial inflammatory insult itself has already fully subsided $(21,22)$. Whether and to 
what extent altered splanchnic nerve signaling correlates with post-inflammatory hypersensitivity remains unclear. Therefore we studied rat splanchnic afferent nerve discharge in response to colorectal distension either in the acute phase of TNBS-colitis or in the post-inflammatory phase. In addition, we assessed whether sensitized responses were associated with visceral hypersensitivity in vivo by quantifying the VMRs to colorectal distension.

Of note, part of this work was presented as an abstract at nEUROgastro 2015 (23).

\section{Material and methods}

\section{Animals}

Adult male Sprague-Dawley rats (200-225g, Charles River, Lecco, Italy) were allowed to acclimatize to housing conditions for one week prior to experimentation and had free access to water and food. All experiments were approved by the Committee for Medical Ethics and the use of Experimental Animals at the University of Antwerp (file number 2010-18).

\section{Colitis}

Distal colitis was induced by the intrarectal instillation of $0.5 \mathrm{~mL}$ of $15 \mathrm{mg}$ TNBS in $50 \%$ ethanol under pentobarbital anesthesia (60 mg. $\mathrm{kg}^{-1} \mathrm{ip}$ ). Control animals received a $0.5 \mathrm{~mL}$ saline enema. The relatively high concentration of ethanol relates to strain differences in susceptibility to TNBS-colitis as previously described (24).

\section{Colonoscopy}

Under pentobarbital anesthesia ( $45 \mathrm{mg} \cdot \mathrm{kg}^{-1} \mathrm{ip}$ ), the lubricated tip of a baby gastroscope (Olympus Europa GmbH, Hamburg, Germany) was gently introduced into the distal colon and advanced under direct endoscopic vision up to $10 \mathrm{~cm}$ proximal to the anal verge. During withdrawal, mucosal damage was assessed using our previously published scoring system $(25,26)$. The colonoscopic score (total 019) takes into account the degree of ulceration (0-6), the extent of damage (0-10) and presence of 
Deiteren et al - 6

edema (0-1), active bleeding (0-1) and/or stenosis (0-1). We previously demonstrated that colonoscopic damage scores correlate highly with the traditional methods to assess TNBS-colitis, namely macroscopic and microscopic scoring systems and myeloperoxidase (MPO) activity (25).

\section{Post-mortem markers of inflammation}

At the end of the in vivo experiments, the distal colon was rapidly excised to evaluate macroscopic mucosal damage using a validated scoring system (score $0-10)(25,27)$. A representative section was fixed in $4 \%$ formaldehyde for $24 \mathrm{~h}$ and embedded in paraffin for hematoxylin-eosin staining. Histological specimens (5 $\mu \mathrm{m}$ slices) were scored microscopically for the presence of an inflammatory infiltrate, the number of infiltrated layers, mucosal architectural distortion and edema (score 0-10) $(25,28)$. A second representative $1 \mathrm{~cm}$ segment was used to assay MPO activity according to our previously published methods (25). In brief, specimens were blotted dry and placed in a potassium phosphate buffer $\mathrm{pH} 6.0$ containing $0.5 \%$ hexadecyltrimethylammonium bromide a rato of $5 \mathrm{~g}$ per $100 \mathrm{~mL}$ buffer. The samples were arranged on ice, homogenized for $30 \mathrm{~s}$ and subjected to two sonication and freeze-thawing cycles. The suspension was centrifuged at $15000 \mathrm{~g}$ for $15 \mathrm{~min}$ at $4{ }^{\circ} \mathrm{C}$. Aliquots $(100 \mu \mathrm{L})$ of the supernatant were added to $2.9 \mathrm{~mL}$ of $o$-dianisidine solution (16.7 $\mathrm{mg}$ of $o$ dianisidine in $1 \mathrm{~mL}$ saline, $98 \mathrm{~mL}$ of $50 \mathrm{mmol}$ potassium phosphate buffer, $\mathrm{pH} 6.0$ and $1 \mathrm{~mL}$ of a 0.05 $\% \mathrm{H}_{2} \mathrm{O}_{2}$ solution as a substrate for MPO enzyme). The change in absorbance was read at $460 \mathrm{~nm}$ over 60 s using a Spectronic Genesys 5 spectrophotometer (Milton Roy, Rochester, NY, USA). One unit of MPO activity was defined as the quantity able to convert $1 \mu$ mol $\mathrm{H}_{2} \mathrm{O}_{2}$ to $\mathrm{H}_{2} \mathrm{O}$ per min at $25^{\circ} \mathrm{C}$ and was expressed as units per gram of tissue $\left(U \cdot g^{-1}\right)$.

\section{Visceromotor responses to colorectal distension}

VMRs to colorectal distension were used as an objective measure of visceral sensitivity. This validated nociceptive reflex consists of the contraction of the abdominal musculature in response to noxious colorectal distension and integrates both peripheral and central processing $(26,28,29)$. 
Other reliable outcome parameters to assess visceral sensitivity in vivo include variations in heart rate and blood pressure or changes in colonic balloon pressure $(29,30)$.

Three days prior to VMR assessment, two EMG electrodes were sutured into the oblique abdominal muscle and were exteriorized at the base of the neck for future access. On the day of VMR assessment, the electrodes were connected to a data acquisition system. A lubricated balloon $(4 \mathrm{~cm}$ length) was gently introduced through the anus and inserted in the colorectum up to $0.5 \mathrm{~cm}$ passed the anal verge. The balloon catheter was secured to the base of the tail and connected to a barostat (Distender Series $\mathrm{II}^{\mathrm{TM}}$ Barostat, G\&J Electronics, Willowdale, Ontario, Canada) for graded and pressure-controlled balloon distension $(10-60 \mathrm{mmHg}, 20 \mathrm{~s}$ duration, $4 \mathrm{~min}$ interval) as previously published (28). The abdominal EMG signal was recorded (NL100AK headstage), amplified (NL104), filtered (NL 125/126, Neurolog, Digitimer Ltd, bandpass 50-5000 Hz) and digitized (CED 1401, Cambridge Electronic Design, Cambridge, UK) to a PC for off-line analysis using Spike2 version 5.16 for Windows (Cambridge Electronic Design). The analog EMG signal was rectified and integrated. To quantify the magnitude of the VMR at each distension pressure, the area under the curve (AUC) during distension (20 s) was corrected for the baseline activity (AUC pre-distension, $20 \mathrm{~s}$ ).

\section{Colonic compliance}

Rats were anesthetized (pentobarbital $\left.45 \mathrm{mg} \cdot \mathrm{kg}^{-1}\right)$ and graded volumes $(0-2.5 \mathrm{~mL}$ ) were applied to the balloon inserted in the colorectum while recording the corresponding intracolonic pressure (31).

\section{Splanchnic afferent nerve activity}

Under deep pentobarbital anesthesia $\left(60 \mathrm{mg} \cdot \mathrm{kg}^{-1}\right)$, the distal colon with the mesenteric bundle in place was excised and transferred to a Sylgard-lined organ chamber. This chamber was maintained at $34^{\circ} \mathrm{C}$ and continuously perfused with oxygenated Krebs solution $(118 \mathrm{mM} \mathrm{NaCl}, 4.75 \mathrm{mM} \mathrm{KCl}, 1 \mathrm{mM}$ $\mathrm{NaH}_{2} \mathrm{PO}_{4}, 22 \mathrm{mM} \mathrm{NaHCO}, 1.2 \mathrm{mM} \mathrm{MgSO}$, $2.5 \mathrm{mM} \mathrm{CaCl}, 11 \mathrm{mM}$ D-glucose, $3 \mu \mathrm{M}$ indomethacin; 10 $\left.\mathrm{mL} \cdot \mathrm{min}^{-1}\right)$. The proximal and distal end of the colon were secured to an in- and outflow port 
Deiteren et al - 8

respectively for intraluminal perfusion with Krebs solution which was maintained at a constant rate of $1 \mathrm{~mL} \cdot \mathrm{min}^{-1}$ via a perfusion syringe pump. Colorectal distension was induced by closure of the outflow while maintaining the inflow. The mesenteric bundle was pinned out at the base of the chamber and the splanchnic nerve was identified at the origin of the inferior mesenteric artery and dissected free. After transection, the myelin sheet was carefully peeled off and the bare nerve bundle was teased into fine strands. One of these was drawn into a suction electrode that was relayed to a data acquisition system to record action potential discharge as previously described (32, 33).

Multi-unit recordings contained action potentials of different amplitude, width and shape. Individual wavemarks were matched to spike templates by a computerized analysis, allowing single-unit discrimination. A signal-noise ratio of at least 2:1 was enforced before spike allocation. All action potential responses were expressed as impulses per second (imp.s ${ }^{-1}, 10 \mathrm{~s}$ bin size). Afferent unit discharge to colorectal distension was quantified by deducting the spontaneous activity from the response to distension. This allowed us to differentiate the spontaneous activity from the actual response to colorectal distension. Based on the firing discharge profile during colorectal distension, units were categorized as low threshold (LT), wide dynamic range (WDR), high threshold (HT) and mechanically insensitive afferents (MIA) as previously published (34-36). First, the firing rate at 20 $\mathrm{mmHg}$ distension is expressed as the percentage of the response at $60 \mathrm{mmHg}$. This parameter, referred to as LT\%, reflects the relative firing at low compared to high distension pressures and was used to further characterize afferent responses based on their functional features as previously published (35). An LT\% of $40 \%$ represents a linear increase in afferent discharge. An LT\% of $>55 \%$, indicates the main increase in afferent firing occurs during limited colorectal distension which is characteristic for LT. An LT\% of $<15 \%$ is characteristic for HT as this implies a disproportionate increase in afferent firing during noxious distension. WDR have an LT\% between 15 and 55 . Finally, MIA are defined as afferent fibers displaying spontaneous activity that does not increase during colonic distension. 


\section{Experimental design}

Two experimental set-ups were performed (Figure 1).

In set-up 1 (acute colitis), rats were randomized to receive saline (control) or TNBS enema. VMRs or splanchnic afferent nerve signaling were evaluated 3 days later during the acute inflammatory phase. In addition, colonoscopy was performed in both TNBS and saline treated rats to confirm the presence of colitis in addition to macroscopic and microscopic evaluation of the tissue and an MPO activity assay. Set-up 2 (post-colitis) focused on visceral sensitivity in the post-inflammatory phase. Rats were administered saline (control) or TNBS (colitis) and the extent of inflammation was verified colonoscopically on day 3 in all rats, similar to set-up 1 . However, from day 10 onwards, convalescence was monitored individually by repeated colonoscopy that was performed every 4 days as described previously (28). If at any time point colonoscopy still showed signs of mucosal inflammation, the animal was allowed to recover further and colonoscopy was repeated every 4 days. If colonoscopy showed complete mucosal healing, the EMG electrodes were implanted and VMR or splanchnic afferent nerve firing were recorded 3 days later. In addition, the postinflammatory status was confirmed using colonoscopic, macroscopic and microscopic evaluation of the colonic tissue in addition to the MPO activity assay.

\section{Data presentation and statistical analysis}

Data are presented as mean \pm sem for $N$, the number of animals and $n$, the number of units. Preliminary analysis indicated that afferent signaling was similar in control rats of the acute set-up and control rats of the post-inflammatory set-up (data not shown). Therefore data from splanchnic nerve firing in controls from the acute and post-inflammatory set-ups were pooled to reduce the number of animals needed. Variables were analyzed using unpaired Student's $t$-test and generalized estimating equations (GEE) followed by least significant difference (LSD) post-hoc test when appropriate. Frequency distributions to compare the proportion of the different afferent fibers was performed using a Chi-square test. Statistical significance was set at $p<0.05$ for all tests. 
Deiteren et al - 10

\section{Results}

VMRs and splanchnic afferent nerve signaling during acute TNBS-colitis

Intrarectal instillation of TNBS resulted in acute colitis on day 3 post-induction characterized by the presence of multiple serpinginous ulcerations in the distal colon and an increased colonoscopic damage score (7.3 \pm 0.3 for TNBS vs $0.0 \pm 0.0$ for control; $N=17-22 ; p<0.001)$. In addition, acute colitis was further confirmed by the macroscopic damage scores $(7.0 \pm 1.3$ vs $0.0 \pm 0.0$ for control; $p<0.001)$, the microscopic damage scores ( $6.0 \pm 0.6$ vs $0.0 \pm 0.0$ for control; $p<0.001)$ and the MPO activity (16.3 \pm 4.3 U. $\mathrm{g}^{-1}$ vs $0.0 \pm 0.0$ for control; $\mathrm{p}<0.001$ ).

VMRs to colorectal distension increased in a pressure-dependent manner (Figure 2A). During acute TNBS-colitis, VMRs were significantly increased compared to controls for the full range of distension pressures, indicating the presence of marked visceral hypersensitivity.

Distending the balloon in the colorectum with fixed volumes of water heightened the intracolonic pressure in a volume-dependent manner, reflecting colonic compliance. Intracolonic pressures increased more rapidly during acute TNBS-colitis, indicating reduced colonic compliance compared to controls (Figure 2B).

Splanchnic afferent nerves of both control and acute TNBS-colitis rats showed irregular spontaneous activity in the absence of colorectal distension (Figure 3A-C). Mechanosensitivity was assessed by performing ramp distension up to $60 \mathrm{mmHg}$, increasing afferent nerve discharge in a pressuredependent fashion (Figure 3A-C). Each nerve bundle contained in the suction electrode consisted of several different units that could be discriminated based on their spike waveform. In total, 57 single units were discriminated in the multi-unit recordings of 12 controls and 60 units were identified in 17 rats with acute TNBS-colitis. On average, $5 \pm 1$ units were discriminated in each multi-unit recording in controls compared to $4 \pm 1$ units in those of rats with acute TNBS-colitis ( $N=12-17$; ns). When afferent firing was normalized for the number of units in each recording, spontaneous activity was not significantly different between controls and acute colitis rats $\left(0.8 \pm 0.1 \mathrm{imp} . \mathrm{s}^{-1}\right.$ for acute colitis vs $0.5 \pm 0.1$ for controls; $\mathrm{N}=12-17 ; \mathrm{p}=0.07)$. In contrast, afferent nerve discharge to colorectal distension 
normalized for the number of units in each recording, was significantly increased during acute TNBScolitis compared to control from a distension pressure of $10 \mathrm{mmHg}$ onwards, indicating the presence of sensitized afferent responses (Figure 3D).

Based on their response to colorectal distension, the 57 units identified in control specimens were identified as $11 \mathrm{LT}, 16 \mathrm{WDR}, 13 \mathrm{HT}$ and $17 \mathrm{MIA}$; the 60 units of TNBS-colitis rats were categorized as 11 LT, 19 WDR, 22 HT and 8 MIA (Figure 4A). The proportion of these four fiber subtypes was significantly different for acute TNBS-colitis compared to control conditions (Chi-square test; $p<0.05$ ). Spontaneous activity of LT and MIA was significantly more vigorous in rats with acute TNBS-colitis compared to controls, whereas WDR and HT units displayed similar spontaneous firing rates in both groups (Table 1). Each of the four different unit subtypes elicited a characteristic response to ramp distension as described previously (Figure 4B-E) (36). LT disproportionally enhanced their firing rate to limited increases in intraluminal pressure and their discharge rapidly saturated at relatively low distension levels (approximately $20 \mathrm{mmHg}$ ) reaching a plateau. In contrast, WDR responded linearly to increasing distension pressures: these fibers were activated by low levels of distension and afferent discharge continued to rise as the intraluminal pressure increased further. HT typically displayed little activity to low distension levels but responded vigorously to noxious distension. Finally, MIA were unresponsive to ramp distension. LT afferent discharge to colorectal distension in acute colitis rats was significantly more vigorous at the plateau phase (from $20 \mathrm{mmHg}$ onwards; Figures 4A). LT, WDR and HT units from rats with acute TNBS-colitis displayed markedly enhanced afferent firing in response to colorectal distension compared to their control counterparts (Figure 4BD). Of note, all afferent responses to colorectal distension were corrected for spontaneous activity.

\section{Splanchnic afferent nerve signaling and VMRs after the resolution of TNBS-colitis}

Three days after the induction of TNBS-colitis, ulcerations were present in all TNBS-instilled rats (colonoscopic score $5.6 \pm 0.4$ for TNBS vs $0.0 \pm 0.0$ for control; $\mathrm{N}=20-23 ; \mathrm{p}<0.001$ ). During 
Deiteren et al - 12

colonoscopic follow-up, TNBS-colitis resolved spontaneously and colonoscopic resolution was present after a median of 14 days (range 10-22 days). Resolution of TNBS-colitis was confirmed by macroscopic ( $0.0 \pm 0.0$ for TNBS vs $0.0 \pm 0.0$ for control) and microscopic evaluation of the colonic tissue ( $0.0 \pm 0.0$ vs $0.0 \pm 0.0$ for control) in addition to similar MPO activity in both groups at the end of the experiment $(0.8 \pm 0.3$ vs $1.1 \pm 0.3$ for control).

After colonoscopically-proven resolution of colitis, VMRs to colorectal distension remained markedly increased in post-colitis rats compared to controls from 20 to $60 \mathrm{mmHg}$ of distension, indicating the presence of post-inflammatory visceral hypersensitivity (Figure 5A). Colonic compliance was similar in both groups (Figure 5B).

In total, 57 single units were discriminated in the recordings from 12 controls (mean $5 \pm 1$ unit per recording) and 56 units were identified in the recordings from 13 rats with colonoscopically-proven resolution of TNBS-colitis (mean $4 \pm 1$ unit per recording; ns). Spontaneous activity, normalized for the number of units in each recording, tended to be higher in the post-colitis group $\left(0.8 \pm 0.1 \mathrm{imp} . \mathrm{s}^{-1}\right.$ for post-colitis vs $0.4 \pm 0.1$ for controls; $\mathrm{N}=12-13 ; \mathrm{p}=0.06$ ) (Figure $6 \mathrm{~B}-\mathrm{C}$ ). Afferent nerve discharge to colorectal distension, normalized for the number of units in each recording, was significantly increased in rats that had recovered from colitis from a distension pressure of $15 \mathrm{mmHg}$ onwards, indicating sensitized afferent nerve responses (Figure 6D).

The 57 single units from control specimens were identified as 11 LT, 16 WDR, $13 \mathrm{HT}$ and $17 \mathrm{MIA}$, whereas the 56 units from post-colitis rats were categorized as $10 \mathrm{LT}, 18 \mathrm{WDR}, 17 \mathrm{HT}$ and $11 \mathrm{MIA}$ (Figure 7A). Although numerically there were less MIA in the preparations of post-colitis rats, in favor of an increased presence of WDR and $H T$, the proportions were not significantly different between control and post-colitis groups $(p=0.2)$. Spontaneous afferent nerve discharge of LT and MIA was more vigorous in post-colitis rats, whereas spontaneous activity of WDR and HT was similar in both groups (table 2). The afferent response to colorectal distension was significantly increased in WDR and HT fibers of rats that had recovered from TNBS-colitis compared to controls (Figure 7B-E). 


\section{Discussion}

In this study we studied whether enhanced splanchnic afferent signaling in vitro is associated with visceral hypersensitivity in vivo during acute TNBS-colitis and in the postinflammatory phase. Based on their discharge profile during colorectal distension, afferent units were categorized as LT, WDR, HT and MIA. During acute TNBS-colitis, spontaneous afferent discharge was significantly increased in LT and MIA, sensitization of LT, WDR and HT was evident as a leftward shift of the afferent response to colorectal distension and VMRs to colorectal distension were increased. In post-colitis rats, hypersensitivity to mechanical gut distension was mainly mediated by WDR and HT with little effect on LT. In addition, LT and MIA continued to display enhanced spontaneous activity after the resolution of TNBS-colitis. Also VMRs remained significantly increased in het post-inflammatory phase.

The characteristic features of visceral nociceptor sensitization include i) increased spontaneous afferent discharge, ii) enhanced response to suprathreshold stimulation, iii) activation by subthreshold stimuli and/or iv) recruitment of silent nociceptors (37). Several of these features were present in our experiments. Firstly, both LT and MIA demonstrated increased afferent discharge in the absence of colorectal distension. These LT most likely correspond to mucosal and muscularmucosal afferents that signal sensory information in the physiological range, for instance on the presence and/or propulsion of fecal matter. Therefore sensitization of LT as evidenced by their enhanced spontaneous discharge (in addition to greater responses to limited levels of distension) could contribute to symptom generation and spontaneous abdominal pain in IBS patients. Also MIA displayed enhanced spontaneous activity. This class of afferent fibers is insensitive to colorectal distension per definition, and therefore does not contribute to the increased mechanosensitivity observed in vivo. Although we cannot fully exclude that some of these fibers may originate from other structures (such as the bladder for example), the enhanced spontaneous activity of these MIA most likely represent thermo- and chemosensitive signaling afferents. Considering the reports of increased spontaneous release of inflammatory and pro-nociceptive mediators in the colonic mucosa 
Deiteren et al - 14

of patients with IBD and IBS, increased activity of such MIA could contribute to symptoms of abdominal pain.

Secondly, enhanced responses to suprathreshold stimulation were evident for LT, WDR and HT fibers during acute TNBS-colitis and sensitization of WDR and HT persisted in the post-inflammatory phase. These findings are in agreement with a previous report of Hughes et al. (6). Using a different set-up in which colonic segments were opened longitudinally and pinned out flat in a recording chamber, Hughes et al. demonstrated predominant involvement of serosal and mesenteric fibers both during acute TNBS-colitis and in the post-inflammatory phase of colitis in C57BL/6 mice (6). Serosal and mesenteric afferents constitute the main fiber types of mouse splanchnic nerves and their peripheral endings are located near the mesenteric attachment (7). They respond to distortion of the outer layers of the colonic wall and levels of stretch into the noxious range $(6,38)$ and therefore most likely correspond to the WDR and HT population in our experiments. In addition, we found evidence of sensitization towards mechanical distension of LT. The data regarding the sensitization of LT fibers are difficult to interpret as during acute TNBS-colitis LT responses were not significantly increased at the lower levels of distension, reaching significance only for the plateau phase ( $20 \mathrm{mmHg}$ onwards). In the post-inflammatory phase of colitis, LT responses were only more vigorous at 40 to $45 \mathrm{mmHg}$ of distension, indicating that this subtype of splanchnic afferents most likely does not contribute significantly to visceral hypersensitivity in the post-colitis rats. Previously, LT slowly-adapting fibers were reported to display enhanced responses to circumferential stretch during acute TNBS-colitis in the guinea pig (39). Similarly to our findings, LT responses recovered to normal once the inflammation resolved. Finally, sensitization of afferent neurons can manifest as a lowering of the activation threshold or through recruitment of silent nociceptors (37). Both would result in a shift of the afferent functional phenotype. During acute TNBS-colitis, we found less MIA in splanchnic afferent recordings in favor of mainly HT and also WDR whereas the proportion of LT remained constant. In the post-inflammatory phase of colitis however, the fiber proportions were no longer significantly different compared to control. Therefore our findings suggest that silent nociceptors, 
most likely MIA, are recruited during acute TNBS-colitis and may revert back into their silent phenotype after the inflammation has subsided. This hypothesis coincides with previous reports that MIA can be sensitized by local exposure to inflammatory mediators and can acquire a mechanosensitivite phenotype (40), however this was not supported by all studies $(8,17)$.

Both during acute TNBS-colitis as in the post-colitis phase, sensitized afferent responses were associated with enhanced VMRs to colorectal distension in vivo. Changes were more pronounced during acute colitis than after the resolution of colitis in both the in vitro and in vivo set-ups. Several reasons may underlie this difference. First, colonic compliance was reduced during acute TNBS-colitis but returned to baseline values after resolution of colitis. Colonic compliance has been shown to account for approximately $25 \%$ of visceral sensations in healthy subjects (41) and therefore may have altered the response profile during acute colitis. However by opting for a pressure-controlled distension of the colon, lower volumes will have been administered to the colon in the acute colitis group versus the control and post-colitis animals to achieve the same distension pressure. Nevertheless, reduced colonic compliance and altered connective tissue matrix and muscle tone will have influenced how colon is distended, which in turn can affect visceral sensation (42). Secondly, during acute colitis excessive amounts of cytokines and mediators among which ATP, protons and bradykinin are released that can act on sensory receptors located at the afferent nerve endings in the wall thus contributing to visceral hypersensitivity $(5,43,44)$. While sensitizing effects can persist long after this 'inflammatory soup' has resolved, it is not surprising that this post-inflammatory visceral hypersensitivity might be of a lesser degree compared to the acute inflammation-induced hypersensitivity.

Of note, in our study, we assessed the splanchnic mechanosensory signaling in relation of visceral hypersensitivity during acute colitis and in the post-inflammatory phase. However, sensory information from the distal colon is conveyed by both the splanchnic and pelvic nerve pathways. 
Deiteren et al - 16

Although traditionally the pelvic nerve is thought to be tuned more towards the transmission of colonic sensations within the physiological range, our group as well as others have demonstrated altered pelvic mechanosensory function in different models of visceral hypersensitivity $(6,9,12-16$, 45). With our study we do not wish to refute or contest the pelvic contribution to visceral hypersensitivity but mainly aimed to highlight the additional importance of splanchnic afferent nerve signaling $(6,8,17,46,47)$.

We have standardized the stimulus in both the in vitro and in vivo set-ups, namely mechanical distension which is the most commonly used stimulus to assess visceral sensory function in human experimental designs (48). In contrast to the flat-sheet preparation, our intact colonic segments do not allow us to identify the location of the afferent ending in the colonic wall (mucosal, muscular, serosal or mesenteric). Nevertheless, segmental distension is a physiological relevant way of demonstrating that these afferents are activated using an integrated stimulus and that sensitized responses contribute toward increased afferent discharge when the gut is distended. In addition we used the same methods and equipment in order to relate our in vitro to in vivo outcomes. Doing so, we demonstrate a good correlation between in vitro electrophysiological recordings and in vivo behavioral assays.

In conclusion, we demonstrated that sensitization of splanchnic primary afferent neurons contributes significantly to visceral hypersensitivity during acute TNBS-colitis and in the post-inflammatory phase of colitis. Spontaneous afferent nerve discharge was increased in LT and MIA and could be associated with increased chemosensitivity. Sensitization to mechanical distension was a prominent feature of LT, WDR and HT fibers during acute colitis and the enhanced afferent discharge of WDR and HT persisted in the post-inflammatory phase. Importantly, we demonstrated that this enhanced afferent discharge to colorectal distension in vitro was correlated with visceral hypersensitivity in vivo as assessed by the VMRs. Although not indicative of a causative relationship, these findings may suggest 
1

2

3

4

5

6

7

8

9

10

11

12

13

14

15

16

17

18

19

20

21

22

23

24

25

26

27

28

29

30

31

32

33

34

35

36

37

38

39

40

41

42

43

44

45

46

47

48

49

50

51

52

53

54

55

56

57

58

59

60

that sensitization of splanchnic afferent nerves is an important factor in the pathogenesis of mechanical visceral hypersensitivity in the TNBS-colitis model. 
7. Acknowledgements, funding, disclosure

Acknowledgements: We would like to thank our lab technicians P. Aerts, A. Jürgens, L. Van Poeck, M. Vinckx and L. R. Van Den Bossche for their technical assistance. A special thanks to dr. David Grundy for his indispensable help in performing the afferent recordings.

Funding: A. Deiteren is an aspirant of the Fund for Scientific Research (FWO), Flanders. This work was supported financially by the FWO (G.0341.13 and G.0249.09N to BDW).

Competing interests: The authors have no competing interests.

Author contribution: $A D$ performed the experiments and wrote the article. $A D, J D M, C K$ and WJ optimized the experimental methods. AD, JDM, CK, WJ, HUDS, PAP, SMF and BYDW provided intellectual contents, extensively revised the article and approved the final version. 
1. Rao SS, Read NW, Davison PA, Bannister JJ, Holdsworth CD. Anorectal sensitivity and responses to rectal distention in patients with ulcerative colitis. Gastroenterology 1987; 93:1270-5.

2. Ritchie J. Pain from distension of the pelvic colon by inflating a balloon in the irritable colon syndrome. Gut 1973; 14:125-32.

3. van Hoboken EA, Thijssen AY, Verhaaren R, van der Veek PP, Prins FA, Verspaget HW, Masclee AA. Symptoms in patients with ulcerative colitis in remission are associated with visceral hypersensitivity and mast cell activity. Scand J Gastroenterol 2011; 46:981-7.

4. Bouin $\mathrm{M}$, Plourde V, Boivin M, Riberdy M, Lupien F, Laganiere M, Verrier P, Poitras P. Rectal distention testing in patients with irritable bowel syndrome: sensitivity, specificity, and predictive values of pain sensory thresholds. Gastroenterology 2002; 122:1771-7.

5. Vermeulen W, De Man JG, Pelckmans PA, De Winter BY. Neuroanatomy of lower gastrointestinal pain disorders. World J Gastroenterol 2014; 20:1005-20.

6. Hughes PA, Brierley SM, Martin CM, Brookes SJ, Linden DR, Blackshaw LA. Post-inflammatory colonic afferent sensitisation: different subtypes, different pathways and different time courses. Gut 2009; 58:1333-41.

7. Brierley SM, Jones RC, 3rd, Gebhart GF, Blackshaw LA. Splanchnic and pelvic mechanosensory afferents signal different qualities of colonic stimuli in mice. Gastroenterology 2004; 127:166-78.

8. Brierley SM, Jones RC, 3rd, Xu L, Gebhart GF, Blackshaw LA. Activation of splanchnic and pelvic colonic afferents by bradykinin in mice. Neurogastroenterol Motil 2005; 17:854-62.

9. Lin C, Al-Chaer ED. Long-term sensitization of primary afferents in adult rats exposed to neonatal colon pain. Brain Res 2003; 971:73-82.

10. Lynn PA, Blackshaw LA. In vitro recordings of afferent fibres with receptive fields in the serosa, muscle and mucosa of rat colon. J Physiol 1999; 518 ( Pt 1):271-82. 
11. Sengupta JN. Visceral pain: the neurophysiological mechanism. Handb Exp Pharmacol 2009:31-74.

12. Feng B, La JH, Schwartz ES, Tanaka T, McMurray TP, Gebhart GF. Long-term sensitization of mechanosensitive and -insensitive afferents in mice with persistent colorectal hypersensitivity. Am J Physiol Gastrointest Liver Physiol 2012; 302:G676-83.

13. Feng B, La JH, Tanaka T, Schwartz ES, McMurray TP, Gebhart GF. Altered colorectal afferent function associated with TNBS-induced visceral hypersensitivity in mice. Am J Physiol Gastrointest Liver Physiol 2012; 303:G817-24.

14. De Schepper HU, De Winter BY, Van Nassauw L, Timmermans JP, Herman AG, Pelckmans PA, De Man JG. TRPV1 receptors on unmyelinated C-fibres mediate colitis-induced sensitization of pelvic afferent nerve fibres in rats. J Physiol 2008; 586:5247-58.

15. Ibeakanma C, Miranda-Morales M, Richards M, Bautista-Cruz F, Martin N, Hurlbut D, Vanner S. Citrobacter rodentium colitis evokes post-infectious hyperexcitability of mouse nociceptive colonic dorsal root ganglion neurons. J Physiol 2009; 587:3505-21.

16. Sengupta JN, Snider A, Su X, Gebhart GF. Effects of kappa opioids in the inflamed rat colon. Pain 1999; 79:175-85.

17. Brierley SM, Carter R, Jones RC, 3rd, Xu L, Robinson DR, Hicks GA, Gebhart GF, Blackshaw LA. Differential chemosensory function and receptor expression of splanchnic and pelvic colonic afferents in mice. J Physiol 2005; 567:267-81.

18. Phillis BD, Martin CM, Kang D, Larsson H, Lindstrom EA, Martinez V, Blackshaw LA. Role of TRPV1 in high-threshold rat colonic splanchnic afferents is revealed by inflammation. Neurosci Lett 2009; 459:57-61.

19. Coldwell JR, Phillis BD, Sutherland K, Howarth GS, Blackshaw LA. Increased responsiveness of rat colonic splanchnic afferents to 5-HT after inflammation and recovery. J Physiol 2007; 579:203-13.

20. Chen J, Winston JH, Sarna SK. Neurological and cellular regulation of visceral hypersensitivity induced by chronic stress and colonic inflammation in rats. Neuroscience 2013; 248C:469-78. 
21. Greenwood-Van Meerveld B, Johnson AC, Foreman RD, Linderoth B. Spinal cord stimulation attenuates visceromotor reflexes in a rat model of post-inflammatory colonic hypersensitivity. Auton Neurosci 2005; 122:69-76.

22. Gschossmann JM, Adam B, Liebregts T, Buenger L, Ruwe M, Gerken G, Mayer EA, Holtmann G. Effect of transient chemically induced colitis on the visceromotor response to mechanical colorectal distension. Eur J Gastroenterol Hepatol 2002; 14:1067-72.

23. Deiteren A, De Man JG, Keating C, Jiang W, Nullens S, Francque SM, Grundy D, De Winter BY. Study of the mechanisms contributing to visceral hypersensitivity in a rat model of acute and postinflammatory colitis: focus on splanchnic afferent nerve signaling. Neurogastroenterol Motil $2015 ; 27: 53$.

24. te Velde AA, Verstege MI, Hommes DW. Critical appraisal of the current practice in murine TNBS-induced colitis. Inflamm Bowel Dis 2006; 12:995-9.

25. Vermeulen W, De Man JG, Nullens S, Pelckmans PA, De Winter BY, Moreels TG. The use of endoscopy to follow the inflammatory time course of TNBS-colitis in rats. Acta Gastroenterol Belg $2011 ; 74: 304-11$.

26. Deiteren A, Vermeulen W, Moreels TG, Pelckmans PA, De Man JG, De Winter BY. The effect of chemically induced colitis, psychological stress and their combination on visceral pain in female Wistar rats. Stress 2014; 17:431-44.

27. Wallace JL, Keenan CM. An orally active inhibitor of leukotriene synthesis accelerates healing in a rat model of colitis. Am J Physiol 1990; 258:G527-34.

28. Deiteren A, De Man JG, Ruyssers NE, Moreels TG, Pelckmans PA, De Winter BY. Histamine H4 and $\mathrm{H} 1$ receptors contribute to postinflammatory visceral hypersensitivity. Gut 2014; 63:1873-82.

29. Ness TJ, Gebhart GF. Colorectal distension as a noxious visceral stimulus: physiologic and pharmacologic characterization of pseudaffective reflexes in the rat. Brain Res 1988; 450:153-69. 
Deiteren et al - 22

30. Tammpere A, Brusberg M, Axenborg J, Hirsch I, Larsson H, Lindstrom E. Evaluation of pseudoaffective responses to noxious colorectal distension in rats by manometric recordings. Pain 2005; 116:220-6.

31. Vermeulen W, De Man JG, De Schepper HU, Bult H, Moreels TG, Pelckmans PA, De Winter BY. Role of TRPV1 and TRPA1 in visceral hypersensitivity to colorectal distension during experimental colitis in rats. Eur J Pharmacol 2013; 698:404-12.

32. Jiang W, Kirkup AJ, Grundy D. Mast cells drive mesenteric afferent signalling during acute intestinal ischaemia. J Physiol 2011; 589:3867-82.

33. Keating C, Pelegrin P, Martinez CM, Grundy D. P2X7 Receptor-Dependent Intestinal Afferent Hypersensitivity in a Mouse Model of Postinfectious Irritable Bowel Syndrome. J Immunol 2011; 187:1467-74.

34. Blackshaw LA, Gebhart GF. The pharmacology of gastrointestinal nociceptive pathways. Curr Opin Pharmacol 2002; 2:642-9.

35. Booth CE, Shaw J, Hicks GA, Kirkup AJ, Winchester W, Grundy D. Influence of the pattern of jejunal distension on mesenteric afferent sensitivity in the anaesthetized rat. Neurogastroenterol Motil 2008; 20:149-58.

36. Keating C, Beyak M, Foley S, Singh G, Marsden C, Spiller R, Grundy D. Afferent hypersensitivity in a mouse model of post-inflammatory gut dysfunction: role of altered serotonin metabolism. J Physiol 2008; 586:4517-30.

37. Burnstock G. Purines and sensory nerves. In: Canning BJ, Spina D, editors. Sensory Nerves. 2009 ed: Springer Berlin Heidelberg; 2009. p. 333-92.

38. Brierley SM, Page AJ, Hughes PA, Adam B, Liebregts T, Cooper NJ, Holtmann G, Liedtke W, et al. Selective role for TRPV4 ion channels in visceral sensory pathways. Gastroenterology 2008; 134:2059-69. 
39. Lynn PA, Chen BN, Zagorodnyuk VP, Costa M, Brookes SJ. TNBS-induced inflammation modulates the function of one class of low-threshold rectal mechanoreceptors in the guinea pig. Am J Physiol Gastrointest Liver Physiol 2008; 295:G862-71.

40. Feng B, Gebhart GF. Characterization of silent afferents in the pelvic and splanchnic innervations of the mouse colorectum. Am J Physiol Gastrointest Liver Physiol 2011; 300:G170-80.

41. Iturrino J, Camilleri M, Busciglio I, Burton D, Zinsmeister AR. Sensations of gas and pain and their relationship with compliance during distension in human colon. Neurogastroenterol Motil 2012; 24:646-51, e275.

42. Larsson M, Arvidsson S, Ekman C, Bayati A. A model for chronic quantitative studies of colorectal sensitivity using balloon distension in conscious mice -- effects of opioid receptor agonists. Neurogastroenterol Motil 2003; 15:371-81.

43. Deiteren A, van der Linden L, de Wit A, Ceuleers H, Buckinx R, Timmermans JP, Moreels TG, Pelckmans PA, et al. P2X3 receptors mediate visceral hypersensitivity during acute chemicallyinduced colitis and in the post-inflammatory phase via different mechanisms of sensitization. PLoS One 2015; 10:e0123810.

44. Peiris M, Bulmer DC, Baker MD, Boundouki G, Sinha S, Hobson A, Lee K, Aziz Q, et al. Human visceral afferent recordings: preliminary report. Gut 2011; 60:204-8.

45. Wynn G, Ma B, Ruan HZ, Burnstock G. Purinergic component of mechanosensory transduction is increased in a rat model of colitis. Am J Physiol Gastrointest Liver Physiol 2004; 287:G647-57.

46. Traub RJ. Evidence for thoracolumbar spinal cord processing of inflammatory, but not acute colonic pain. Neuroreport 2000; 11:2113-6.

47. Traub RJ, Murphy A. Colonic inflammation induces fos expression in the thoracolumbar spinal cord increasing activity in the spinoparabrachial pathway. Pain 2002; 95:93-102. 
48. Keszthelyi D, Troost FJ, Masclee AA. Irritable bowel syndrome: methods, mechanisms, and pathophysiology. Methods to assess visceral hypersensitivity in irritable bowel syndrome. Am J Physiol Gastrointest Liver Physiol 2012; 303:G141-54. 
1

2

3

4

5

6

7

8

9

10

11

12

13

14

15

16

17

18

19

20

21

22

23

24

25

26

27

28

29

30

31

32

33

34

35

36

37

38

39

40

41

42

43

44

45

46

47

48

49

50

51

52

53

54

55

56

57

58

59

60

\section{Abbreviations}
AUC area under the curve
DSS dextran sulphate sodium
GEE generalized estimating equations
HT high threshold
IBD inflammatory bowel disease
IBS irritable bowel syndrome
LSD least significant difference
LT low threshold
MIA mechanically insensitive afferent
MPO myeloperoxidase
TNBS trinitrobenzene sulphonic acid
VMR visceromotor response
WDR wide dynamic range 


\section{Tables}

Table 1. Spontaneous firing rate of single units in controls and rats with acute TNBS-colitis.

\begin{tabular}{lll}
\hline & $\begin{array}{l}\text { Control } \\
\left(\text { imp. } s^{-1}\right)\end{array}$ & Colitis \\
& $0.3 \pm 0.1$ & $1.2 \pm 0.3^{*}$ \\
LT & $0.6 \pm 0.2$ & $0.9 \pm 0.1$ \\
WDR & $0.6 \pm 0.2$ & $0.6 \pm 0.2$ \\
HT & $0.4 \pm 0.1$ & $1.1 \pm 0.1^{* *}$ \\
MIA & & \\
\hline
\end{tabular}

Results expressed as impulses per second and presented as mean \pm sem $n=8-22$. LT, low threshold; WDR, wide dynamic range; $H T$, high threshold; MIA, mechanically insensitive afferents. Unpaired Student's $t$ test; ${ }^{*} p<0.05,{ }^{* *} p<0.01$, significantly different from control. 
1

2

3

4

5

6

7

8

9

10

11

12

13

14

15

16

17

18

19

20

21

22

23

24

25

26

27

28

29

30

31

32

33

34

35

36

37

38

39

40

41

42

43

44

45

46

47

48

49

50

51

52

53

54

55

56

57

58

59

60

Table 2. Spontaneous firing rate of single units in controls and rats that had recovered from TNBS-colitis.

\begin{tabular}{lll}
\hline & $\begin{array}{c}\text { Control } \\
\left(\text { imp. } .^{-1}\right)\end{array}$ & $\begin{array}{l}\text { Post-colitis } \\
\left(\text { imp. } .^{-1}\right)\end{array}$ \\
\hline LT & $0.3 \pm 0.1$ & $0.8 \pm 0.3^{*}$ \\
WDR & $0.6 \pm 0.2$ & $0.8 \pm 0.2$ \\
HT & $0.6 \pm 0.2$ & $0.4 \pm 0.1$ \\
MIA & $0.4 \pm 0.1$ & $1.0 \pm 0.2^{*}$ \\
\hline
\end{tabular}

Results expressed as impulses per second and presented as mean \pm sem for $\mathrm{N}=12-13$ and $\mathrm{n}=10-18$. LT, low threshold; WDR, wide dynamic range; HT, high threshold; MIA, mechanically insensitive afferents. Unpaired Student's $t$-test; ${ }^{*} p<0.05$, significantly different from control. 
Deiteren et al - 28

\section{Figures legends}

Figure 1. Schematic diagram of the experimental design. In set-up 1 (acute colitis), rats were treated with TNBS (colitis) or saline (control) and visceral sensitivity was assessed 3 days later by quantifying VMRs and splanchnic afferent nerve discharge to colorectal distension. In set-up 2, rats were treated with TNBS or saline and the extent and healing process of colitis was monitored individually by repeated colonoscopy: first on day 3 to confirm the presence of colitis and thereafter, starting from day 10 , every 4 days until complete mucosal healing (score $=0$ ) had occurred. Visceral sensitivity was evaluated 3 days after colonoscopic mucosal healing by quantifying VMRs and splanchnic afferent nerve discharge to colorectal distension. In both set-up 1 and set-up 2, the inflammatory status was assessed by colonoscopic, macroscopic and microscopic evaluation of the colonic tissue in addition to a myeloperoxidase activity assay. TNBS, trinitrobenzene sulphonic acid; VMR, visceromotor response.

Figure 2. The effect of acute TNBS-colitis (filled squares) compared to controls (open triangles) on visceromotor responses (VMRs) to colorectal distension (A) and colonic compliance (B). Generalized estimating equations, LSD post-hoc test; $\mathrm{N}=5$ /group; ${ }^{*} \mathrm{p}<0.05, * * \mathrm{p}<0.01, * * * \mathrm{p}<0.001$, significantly different compared to controls (open triangles).

Figure 3. The effect of acute TNBS-colitis on spontaneous and distension-induced splanchnic afferent nerve firing. Closure of the segmental outflow while maintaining the inflow resulted in colonic ramp distension (A). Representative tracings of spontaneous and distension-induced afferent discharge are shown in B and C respectively. Afferent firing during colorectal distension (D) was corrected for spontaneous activity, normalized to the number of units in each recording and expressed as impulses per second (imp/s). Generalized estimating equations, LSD post-hoc test; N=12-17/group; * $p<0.05$, $* * \mathrm{p}<0.01, * * * \mathrm{p}<0.001$, significantly different compared to controls. 
Figure 4. The proportion of $L T, W D R, H T$ and MIA in recordings from 12 control rats and 17 rats with acute TNBS-colitis. Chi-square test for $n=57-60 ;{ }^{*} p<0.05$, proportions significantly different compared to controls (A). Afferent firing to colorectal distension for LT, WDR, HT and MIA are shown in B, C, D and E respectively. Afferent firing was corrected for spontaneous activity. Generalized estimating equations, LSD post-hoc test; $\mathrm{n}=10$-22/group; ${ }^{*} \mathrm{p}<0.05, * * \mathrm{p}<0.01,{ }^{* * *} \mathrm{p}<0.001$, significantly different compared to controls. LT, low threshold; WDR, wide dynamic range; HT, high threshold; MIA, mechanically insensitive afferents.

Figure 5. Visceromotor responses (VMRs) to colorectal distension (A) and colonic compliance (B) in control rats (open triangles) and rats with colonoscopically-proven resolution of TNBS-colitis (filled circles). Generalized estimating equations, LSD post-hoc test; $\mathrm{N}=5-10 /$ group $^{*} \mathrm{p}<0.05,{ }^{* *} \mathrm{p}<0.01,{ }^{* * *}$ $p<0.001$, significantly different compared to controls.

Figure 6. Spontaneous and distension-induced splanchnic afferent nerve firing in control rats and rats with colonoscopically-proven resolution of TNBS-colitis. Closure of the segmental outflow while maintaining the inflow resulted in colonic ramp distension (A). Representative tracings of spontaneous and distension-induced afferent discharge are shown in B and C respectively. Afferent firing during colorectal distension (D) was corrected for spontaneous activity, normalized to the number of units in each recording and expressed as impulses per second (imp/s). Generalized estimating equations, LSD post-hoc test; $\mathrm{N}=12-13$ /group; * $\mathrm{p}<0.05,{ }^{* *} \mathrm{p}<0.01, * * * \mathrm{p}<0.001$, significantly different compared to controls.

Figure 7. The proportion of $L T, W D R, H T$ and MIA in recordings from 12 control rats and 13 rats with colonoscopically-proven resolution of TNBS-colitis. Chi-square test for $n=56-57$; no significant difference (A). Afferent firing to colorectal distension for LT, WDR, HT and MIA are shown in B, C, D and $\mathbf{E}$ respectively. Afferent firing was corrected for spontaneous activity. Generalized estimating 
Deiteren et al - 30

equations, LSD post-hoc test; $\mathrm{n}=10-18 ;{ }^{*} \mathrm{p}<0.05,{ }^{* *} \mathrm{p}<0.01, * * * \mathrm{p}<0.001$, significantly different compared to controls. LT, low threshold; WDR, wide dynamic range; HT, high threshold; MIA, mechanically insensitive afferents. 
Figure 1. Schematic diagram of the experimental design. In set-up 1 (acute colitis), rats were treated with TNBS (colitis) or saline (control) and visceral sensitivity was assessed 3 days later by quantifying VMRs and splanchnic afferent nerve discharge to colorectal distension. In set-up 2, rats were treated with TNBS or saline and the extent and healing process of colitis was monitored individually by repeated colonoscopy: first on day 3 to confirm the presence of colitis and thereafter, starting from day 10, every 4 days until complete mucosal healing (score $=0$ ) had occurred. Visceral sensitivity was evaluated 3 days after colonoscopic mucosal healing by quantifying VMRs and splanchnic afferent nerve discharge to colorectal distension. In both set-up 1 and set-up 2, the inflammatory status was assessed by colonoscopic, macroscopic and microscopic evaluation of the colonic tissue in addition to a myeloperoxidase activity assay. TNBS, trinitrobenzene sulphonic acid; VMR, visceromotor response. $201 \times 96 \mathrm{~mm}(300 \times 300 \mathrm{DPI})$ 
A)

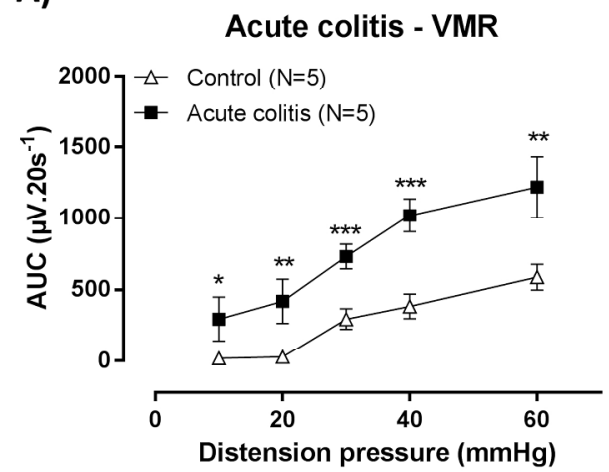

B)

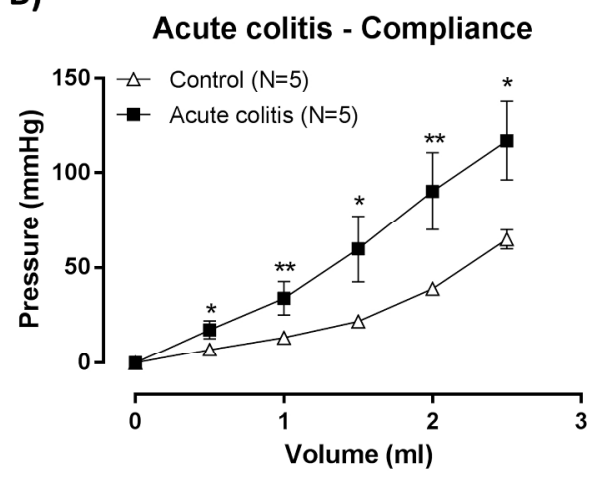

Figure 2. The effect of acute TNBS-colitis (filled squares) compared to controls (open triangles) on visceromotor responses (VMRs) to colorectal distension (A) and colonic compliance (B). Generalized estimating equations, LSD post-hoc test; $\mathrm{N}=5 /$ group; $* \mathrm{p}<0.05$, $* * \mathrm{p}<0.01, * * * \mathrm{p}<0.001$, significantly different compared to controls (open triangles).

$207 \times 83 \mathrm{~mm}(300 \times 300$ DPI) 
Figure 3. The effect of acute TNBS-colitis on spontaneous and distension-induced splanchnic afferent nerve firing. Closure of the segmental outflow while maintaining the inflow resulted in colonic ramp distension ( $A$ ). Representative tracings of spontaneous and distension-induced afferent discharge are shown in B and C respectively. Afferent firing during colorectal distension (D) was corrected for spontaneous activity, normalized to the number of units in each recording and expressed as impulses per second (imp/s). Generalized estimating equations, LSD post-hoc test; $\mathrm{N}=12-17 /$ group; $* \mathrm{p}<0.05, * * \mathrm{p}<0.01, * * * \mathrm{p}<0.001$, significantly different compared to controls.

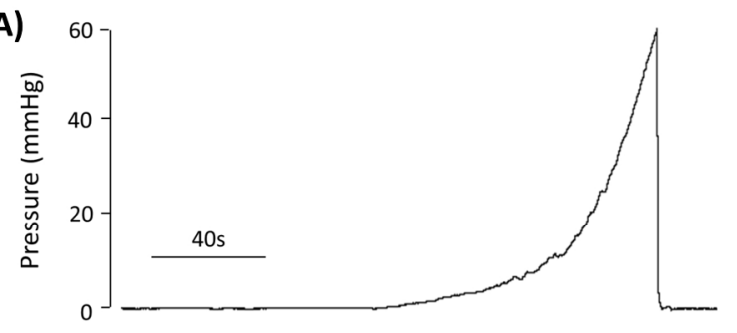

B) 5007 Control
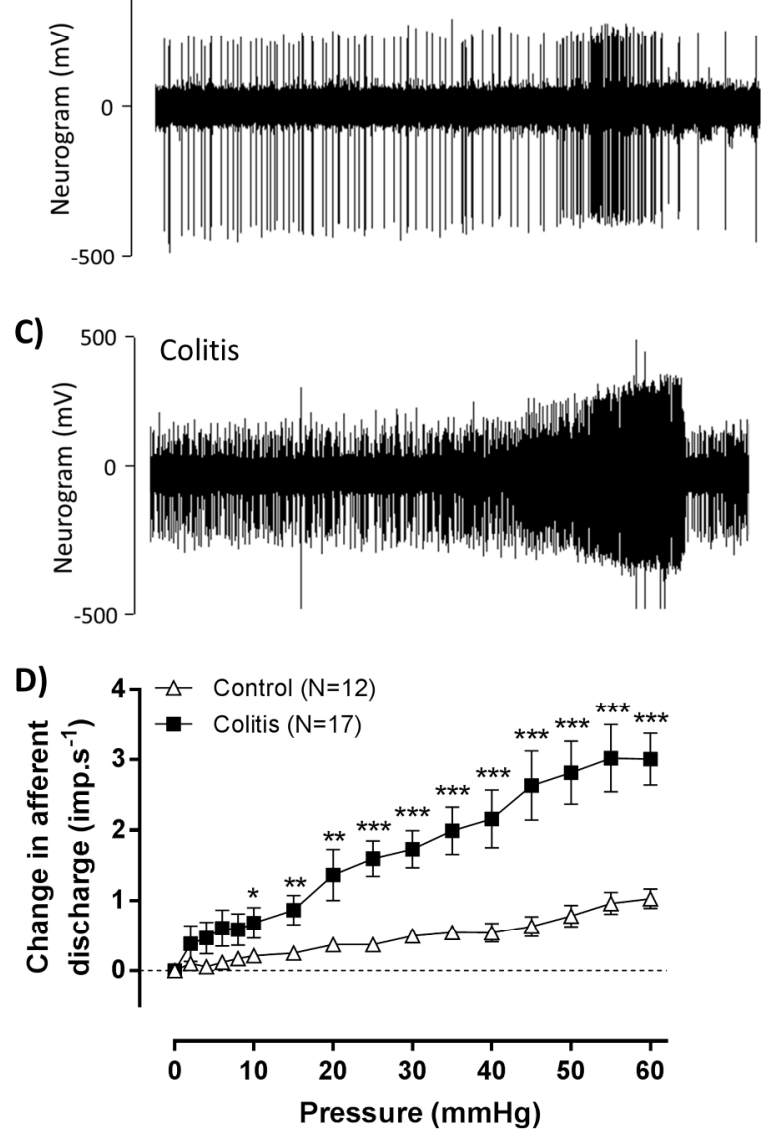

\section{$115 \times 234 \mathrm{~mm}$ (300 x 300 DPI)}


A)

\section{Control}

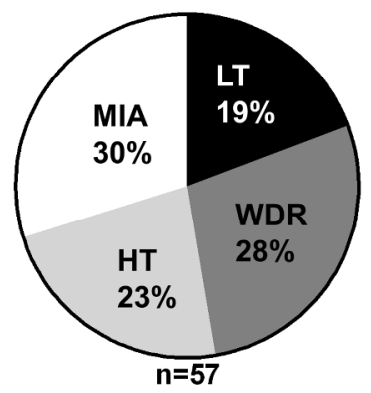

B)

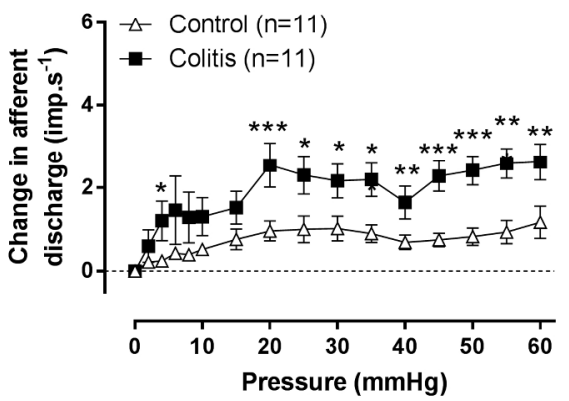

D)

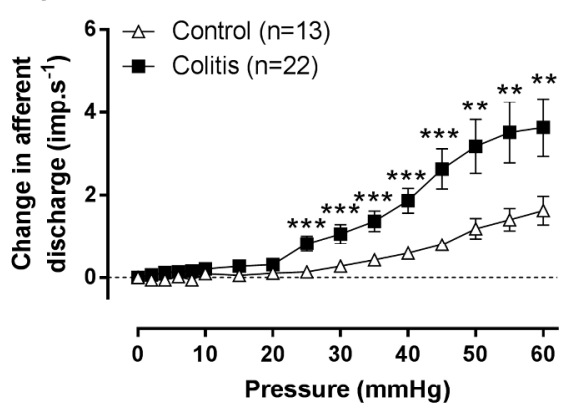

Colitis *

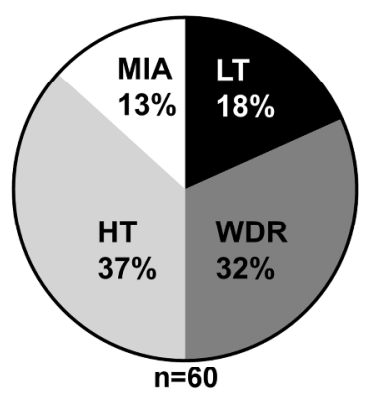

C)

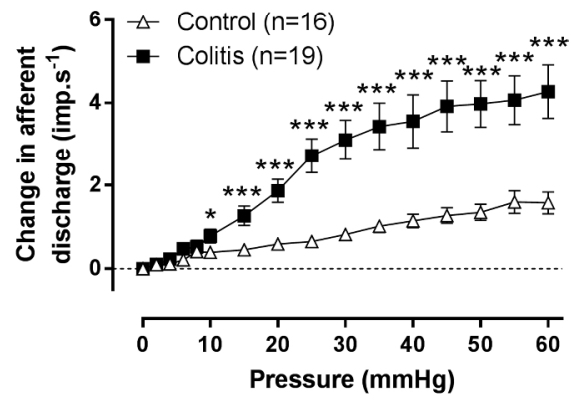

E)

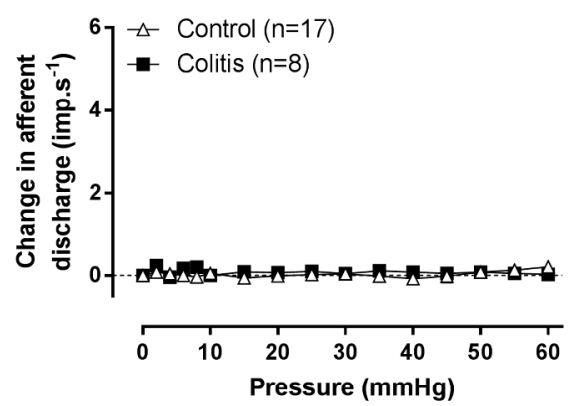

Figure 4. The proportion of LT, WDR, HT and MIA in recordings from 12 control rats and 17 rats with acute TNBS-colitis. Chi-square test for $\mathrm{n}=57-60 ; * \mathrm{p}<0.05$, proportions significantly different compared to controls (A). Afferent firing to colorectal distension for LT, WDR, HT and MIA are shown in B, C, D and E respectively. Afferent firing was corrected for spontaneous activity. Generalized estimating equations, LSD post-hoc test; $\mathrm{n}=10-22 /$ group; $* \mathrm{p}<0.05, * * \mathrm{p}<0.01, * * * \mathrm{p}<0.001$, significantly different compared to controls. LT, low threshold; WDR, wide dynamic range; HT, high threshold; MIA, mechanically insensitive afferents. 
A)

Post-colitis - VMR

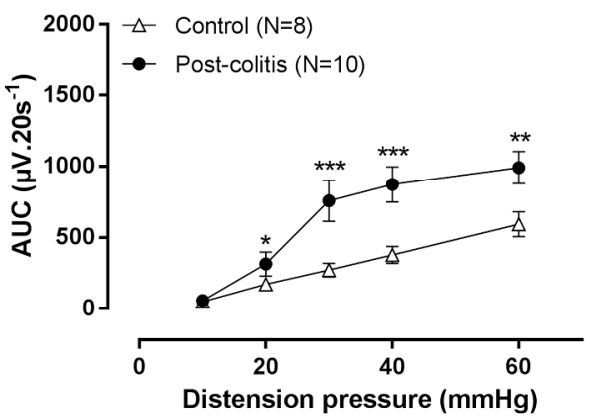

B)

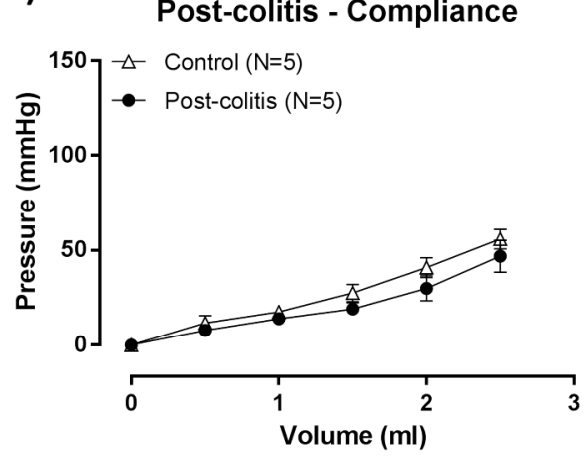

Figure 5. Visceromotor responses (VMRs) to colorectal distension (A) and colonic compliance (B) in control rats (open triangles) and rats with colonoscopically-proven resolution of TNBS-colitis (filled circles). Generalized estimating equations, LSD post-hoc test; $\mathrm{N}=5$-10/group; $* \mathrm{p}<0.05, * * \mathrm{p}<0.01, * * * \mathrm{p}<0.001$, significantly different compared to controls. $209 \times 81 \mathrm{~mm}(300 \times 300 \mathrm{DPI})$ 

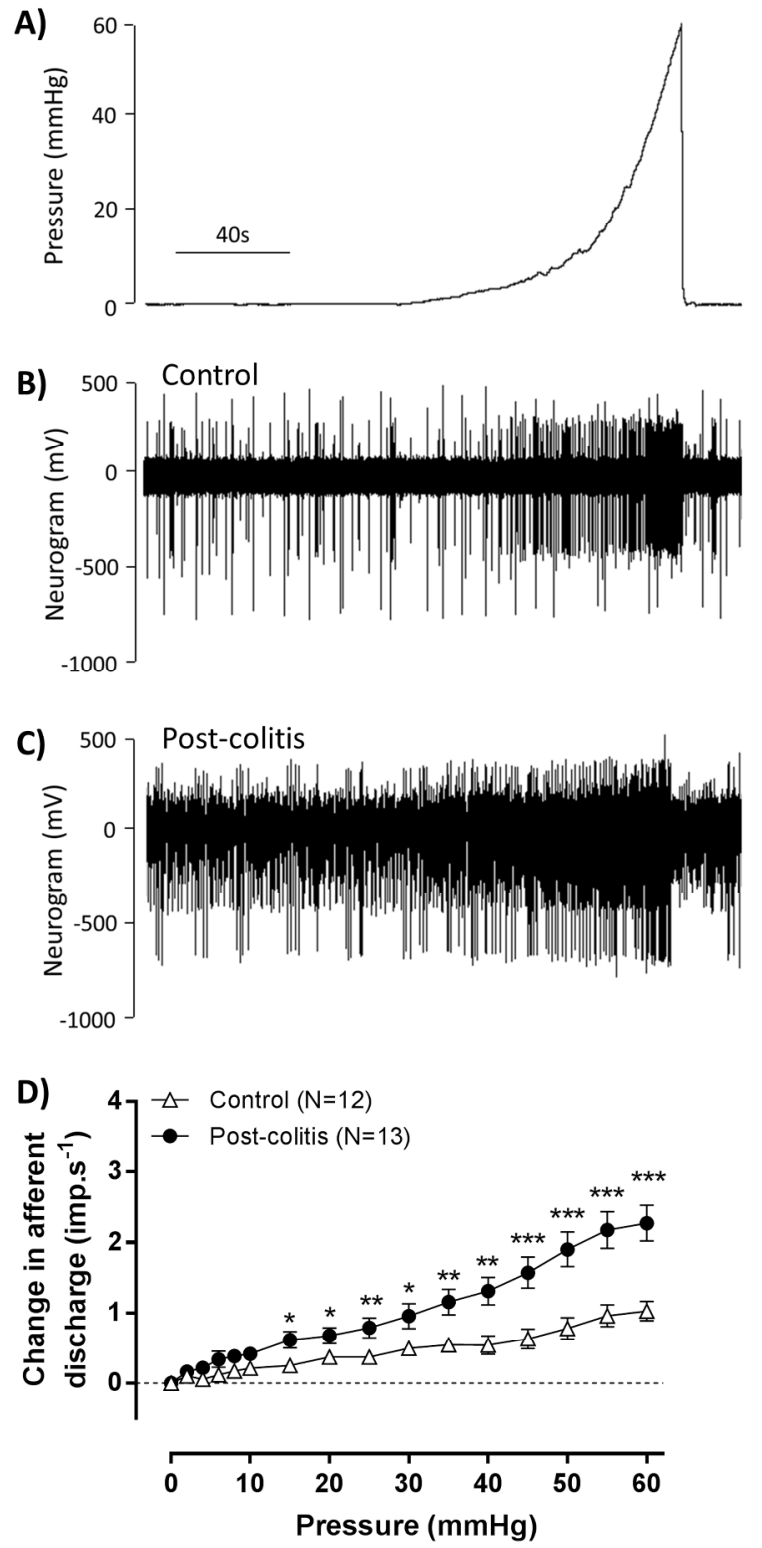

Figure 6. Spontaneous and distension-induced splanchnic afferent nerve firing in control rats and rats with colonoscopically-proven resolution of TNBS-colitis. Closure of the segmental outflow while maintaining the inflow resulted in colonic ramp distension (A). Representative tracings of spontaneous and distensioninduced afferent discharge are shown in $B$ and $C$ respectively. Afferent firing during colorectal distension (D) was corrected for spontaneous activity, normalized to the number of units in each recording and expressed as impulses per second (imp/s). Generalized estimating equations, LSD post-hoc test; N=12-13/group; * $\mathrm{p}<0.05, * * \mathrm{p}<0.01, * * * \mathrm{p}<0.001$, significantly different compared to controls. 
A)

\section{Control}

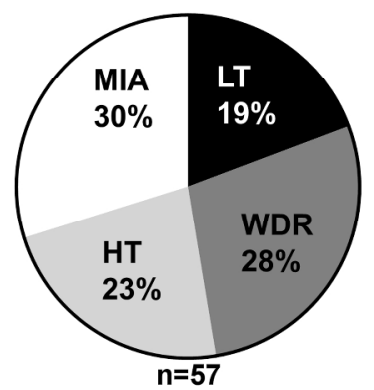

B)

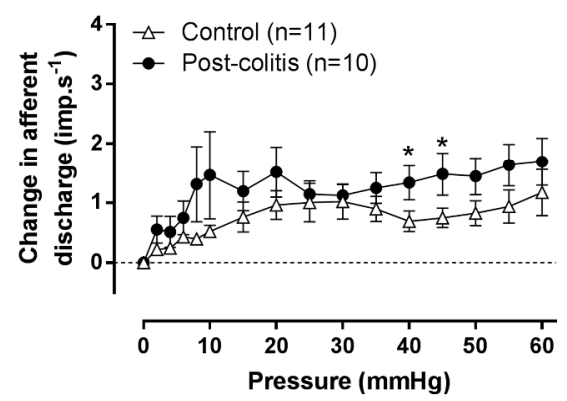

D)

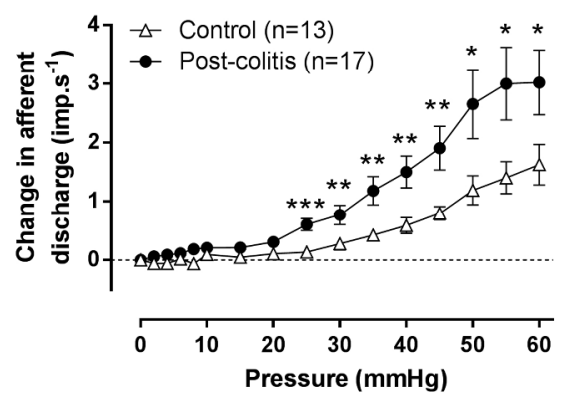

Post-colitis

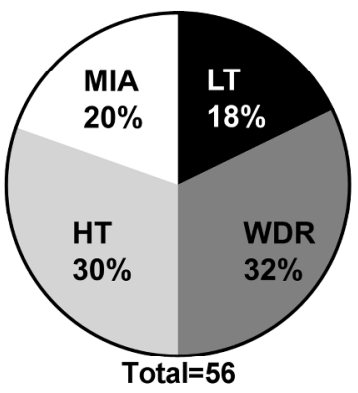

C)

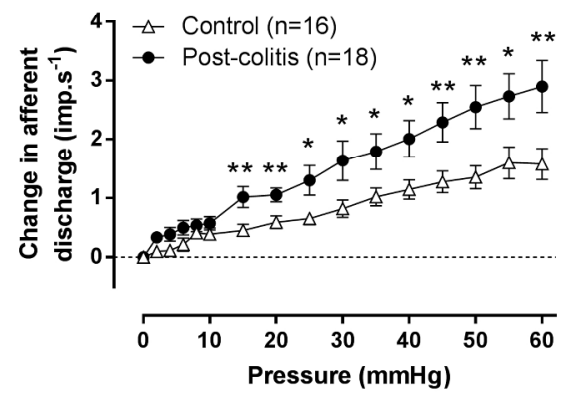

E)

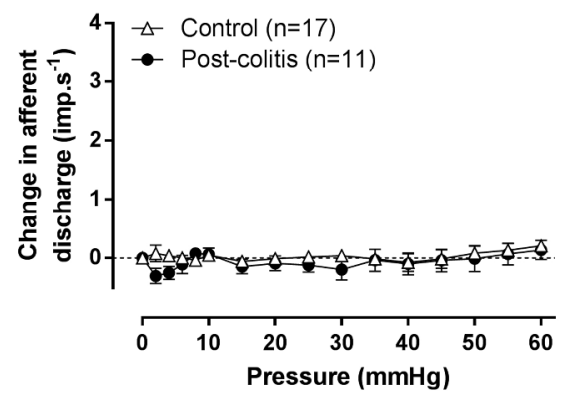

Figure 7. The proportion of LT, WDR, HT and MIA in recordings from 12 control rats and 13 rats with colonoscopically-proven resolution of TNBS-colitis. Chi-square test for $n=56-57$; no significant difference (A). Afferent firing to colorectal distension for LT, WDR, HT and MIA are shown in B, C, D and E respectively. Afferent firing was corrected for spontaneous activity. Generalized estimating equations, LSD post-hoc test; $\mathrm{n}=10-18 ; * \mathrm{p}<0.05, * * \mathrm{p}<0.01, * * * \mathrm{p}<0.001$, significantly different compared to controls. LT, low threshold; WDR, wide dynamic range; HT, high threshold; MIA, mechanically insensitive afferents. 
We are very pleased that the editorial board has shown interest in our manuscript entitled

"Mechanisms contributing to visceral hypersensitivity: focus on splanchnic afferent nerve signaling". We thank you and the two expert referees for the valuable and positive comments. In accord with their suggestions we have revised the manuscript to address their concerns. Please find below a detailed point-to-point reply to the comments that were raised. We hope we addressed the questions satisfactorily.

Response to the editor

Part of this work was presented at nEUROgastro 2015 [1], sponsored by the European Neurogastroenterology and Motility Society, as now stated at the end of the introduction, page 5 of the revised manuscript.

\section{Response to reviewer 1}

1. The authors need to explicitly describe in this submission how low threshold, WDR and HT afferents are characterized from one another. This is crucial as the authors are reporting changes in the proportion of these afferent subtypes in the colitis model.

Based on the firing discharge profile during colorectal distension, units were categorized as low threshold (LT), wide dynamic range (WDR), high threshold (HT) and mechanically insensitive afferents (MIA) as previously published [2-4]. First, the firing rate at $20 \mathrm{mmHg}$ distension is expressed as the percentage of the response at $60 \mathrm{mmHg}$. This parameter, referred to as LT\%, reflects the relative firing at low compared to high distension pressures and was used to further characterize afferent responses based on their functional features as previously published [3]. An LT\% of 40\% represents a linear increase in afferent discharge. An LT\% of $>55 \%$, indicates the main increase in afferent firing occurs during limited colorectal distension which is characteristic for LT. An LT\% of $<15 \%$ is characteristic for HT as this implies a disproportionate increase in afferent firing during noxious distension. WDR have an LT\% between 15 and 55. Finally, MIA are defined as afferent fibers displaying spontaneous activity that does not increase during colonic distension.

This is described in the revised manuscript on page 8.

2. In addition, MIA afferents have been defined as 'afferent fibers displaying spontaneous activity but do not increase during colonic distension'. However, depending on exactly where the recordings are performed from this firing is not necessarily indicative of an afferent innervating the colon. If the recording is the spinal side of the inferior mesenteric ganglion it could in fact be an afferent innervating another organ (bladder for example). This needs to be clarified by the authors. We agree with the reviewer that although we dissected the tissue in such a way that only the colon and a minimum of mesenteric tissue was present, we cannot exclude that some non-mechanosensitive afferent firing to relate to innervation of other tissue beside the colon.

This is now included in the discussion of the revised manuscript on page 13-14.

3. The authors should discuss why they used a relatively high concentration of ethanol (50\%) in their TNBS studies when other studies have used lower ethanol concentrations. This may be a simply issue of comparing rats vs. mice but it warrants a comment for comparative purposes.

Indeed, the concentration of ethanol is relatively high. However, we found this dose of TNBS (15 mg) in combination with $50 \%$ of ethanol optimal to induce a reproducible degree of colitis in our SpragueDawley rats. This is higher compared to the dose and concentration we previously used in Wistar rats (7.5 mg of TNBS in 40\% of ethanol) [5] and Swiss mice (10 mg of TNBS in 30\% ethanol) [6] and most likely relates to species and strain differences in the susceptibility to TNBS-induced colitis as was previously discussed by others [7].

A comment on this was inserted in the methods section of the revised manuscript on page 5 .

4. The authors have measured colonic compliance in the control, acute colitis and post-colitis animals. Notably, they show that compliance is only altered in acute colitis and not in the post-colitis animals. However, they make no point of comment in the discussion on how this may alter their findings. This should be addressed, as the altered compliance is likely to alter the response profiles in the acute colitis animals. Essentially there is a leftward shift in the pressure curve vs. volume and the 
authors will actually be administering higher pressures at any given volume in the acute colitis vs the control and post-colitis animals. This will have a direct effect on afferent sensitivity and may explain some of their alterations in afferent and VMR responses. This should be clarified and commented on in the discussion.

Indeed, colonic compliance was reduced in the acute colitis setting compared to control and postcolitis rats as evidenced by a leftward shift of the pressure-volume curve. In a volume-controlled setting this would indeed mean that higher pressures are administered at any given volume in the acute colitis vs the control and post-colitis animals. However, as we have opted for a pressurecontrolled system, this means that to achieve the same distension pressures lower volumes will be administered in the acute colitis versus the control and post-colitis animals. Nevertheless, reduced colonic compliance and altered connective tissue matrix and muscle tone will have influenced how colon is distended, which in turn can affect visceral sensation [8]. This paragraph is now included in the discussion of the revised manuscript (page 15-16).

5. Discussion. Page 14 Line 18. The authors statement starting with "However it remains uncertain whether distending a segment to $60 \mathrm{mmHg}$ is a large enough stimulus to activated these mesenteric/serosal afferents".... and continues until line 34. The authors should read "Selective Role for TRPV4 Ion Channels in Visceral Sensory Pathways" by Brierley et al., 2008 Gastroenterology and adjust their conclusion accordingly. This paper compares distension in intact preparations, vs. flat sheet von frey hair and stretch responses. This would suggest that that these afferents are indeed activated by the various stimuli at quite specific mechanical stimulation intensities in the noxious range. If the authors accordingly altered their comments based on these finding, it would actually help to explain their own results.

We thank the reviewer for the suggestion. In the paper of Brierley et al, it was shown that colonic mechanoreceptors with receptive fields on serosa and mesentery responded to high intensities of mechanical stimulation with von Frey hairs ( $>50 \%$ above $400 \mathrm{mg}$ ). This was correlated with a high threshold to distention of the intact colon by balloon ( $45 \pm 3.2 \mathrm{mmHg}$ ) or circumferential stretch of the preparation as a flat sheet $(10.6 \pm 0.3 \mathrm{~g})$ indicating that indeed $60 \mathrm{mmHg}$ is a large enough stimulus to activate mesenteric/serosal afferents.

The paragraph was adjusted accordingly (page 14 of the revised manuscript).

\section{Response to reviewer 2}

1. This paper represents a solid piece of work from a solid group with a good reputation in their field. The methods are well-established and the interpretation is spot on up to a point. The point at which interpretation becomes too speculative is unfortunately the whole point of the study, which is to determine the importance of the splanchnic pathway in visceral pain. What the authors do is to correlate changes in splanchnic afferent fiber mechanosensitivity with changes in behavioral responses to mechanical stimuli. It is commendable that they have standardized the stimulus in both cases, which has not been done before. The problem is they have done nothing to prove that the splanchnic pathway actually mediates the behavioral response. To do this they would have to lesion the nerves somehow and measure VMR before and after. If they can do this and add it to the paper it would make a massive difference to its impact.

It is clear in several species that the splanchnic afferent innervation extends as far as the distal colon, whereupon the pelvic pathway takes over, with a small area of overlap of the two. The group of NJ Spencer has shown that the VMR to rectal distension is abolished by rectal nerve section (which are branches of the pelvic nerve). This is not at all surprising. Similarly I would expect that responses to distension of the distal colon would be abolished by section of the lumbar colonic or splanchnic nerves. It is all a simple matter of anatomy. What is the important question is the pathway of pain in the clinic and/or the pathway of pain in response to a particular type of stimulus. To this end it is critical to know where is the balloon relative to the two sources of innervation.

I think the authors need to either do the extra experiments to determine the contribution of the splanchnic nerve, or change their conclusions substantially to make it clear that they are seeing a correlation, not a causative relationship, between splanchnic afferent activation and VMR. 
The paper is largely in agreement with others on effects of inflammation on sensory pathways. Thus its uniqueness in its current form is performing VMR and afferent studies using the same methods and equipment, which makes it easier to correlate the two. This needs to be better advertised instead of the current conclusions which are too bold.

We agree with the reviewer that we might have been too bold in drawing conclusions from our data as it is indeed a correlation or association that we see between the afferent recordings and the visceromotor responses, which by itself does not suggest a causative relationship. The idea of leasioning the splanchnic nerve in vivo followed by VMR assessment is intriguing. Indeed, in the paper of Nick Spencer's group, referred to by the reviewer, transection of the rectal nerves abolished VMRs to rectal distension [9]. In the same paper, the authors described that similarly leasioning the lumbar nerves did not affect VMRs to rectal distension in healthy control mice under general anesthesia. It would be interesting to evaluate the effect of splanchnic afferent nerve transection on the VMRs in our model to determine the exact contribution of this sensory pathway to the visceral hypersensitivity. However, there are some difficulties in performing these experiments. First, the surgery is quite challenging as the splanchnic afferents are located deep in the abdomen close to the aorta, however this is only a matter of practice. Nevertheless, to perform these experiments in sufficient number of rats in the three experimental conditions (control, acute colitis and post-colitis) is almost a completely new project by itself. Secondly, a major difference between the methodology of the Spencer group and our approach is that we assess VMRs in non-sedated, fully awake rats, as anesthetics are known to significantly alter or even abolish VMRs [10]. This makes it nearly impossible to transect the splanchnic nerve in vivo and afterwards perform VMR assessments as effects due to the surgery itself such as post -operative ileus and other motility dysfunctions due to severing the efferent pathways will undoubtedly impede correct interpretation of the results [11]. Also, transecting the splanchnic nerve will impact bladder function, which due to the crosstalk between bladder and colon might also affect colonic sensitivity as previously published [12]. Finally, there is the aspect of postoperative pain as you open the abdominal muscles and go deep into the abdominal cavity which could affect afferent function.

Therefore, although we agree that these experiments would be highly interesting, we decided to follow the reviewer's advice of revising our conclusions as currently we have insufficient evidence to demonstrate a causative relationship and instead focus on the correlation between splanchnic afferent activation and VMR.

This is now altered in the abstract and throughout the discussion.

Regarding the comment of the reviewer on where the balloon is relative to the splanchnic and pelvic innervation we believe that with our balloon distension in vivo we stimulate both nervous pathways as the balloon covers the segment of the colon that is dissected for the in vitro electrophysiological recordings as can been seen in the picture below. However, we fully acknowledge that this is purely speculative.

2. It is important to note that the VMR has some major weaknesses when it comes to correlation with pain. How much is an effort to expel the balloon and how much is a wincing response to pain is very difficult to say. The fact that the response can be evoked by low intensity stimuli suggests it is not all pain-related. The group led by Larsson investigated this a few years ago, showing that blood pressure and heart rate are much better indices of pain perception. Just because VMR has been used extensively in this field does not mean it is the gold standard. It would be good to see discussion of these aspects.

It is true that although the VMR remains the most commonly used method to assess visceral hypersensitivity to colorectal distension in vivo other techniques are available such as using telemetry to monitor heart rate and blood pressure during distensions. Both the cardiovascular and visceromotor responses act via brainstem loops and are reduced or abolished by classis analgesics among which morphine [10]. There are few papers that compared VMRs and cardiovascular 
responses head-to-head. As the reviewer already mentioned, the group of Larsson published several papers evaluating the antinoceptive potential of among others baclofen and pregabalin on visceral pain looking at VMR, blood pressure and heart rate simultaneously $[13,14]$. However, in their discussion they state that 'the VMR is most sensitive [to inhibition] when compared to cardiovascular responses'. They hypothesize that this 'might point toward the VMR being more tightly controlled by nociceptive input, with cardiovascular responses to colorectal distension being affected by other factors such as emotional state' [13]. Therefore, we are not sure which paper of the group of Larsson the reviewer refers to. Nevertheless, we fully acknowledge that the VMR is not the only technique to assess visceral sensitivity, nor did we intend to claim that it is the gold standard. However, as a discussion on the different methods to assess visceral hypersensitivity is beyond the scope of our manuscript, we hope we comply to the concern of the referee by referring to alternative techniques to assess visceral hypersensitivity in vivo in the methods section on page 7.

3. The discussion of the paper is too long, and strays often from the subject onto matters unrelated to this study.

The discussion has been extensively reworked to address the reviewer's remark.

\section{REFERENCE LIST}

1. Deiteren A, De Man JG, Keating C, Jiang W, Nullens S, Francque SM, et al. (2015) Study of the mechanisms contributing to visceral hypersensitivity in a rat model of acute and postinflammatory colitis: focus on splanchnic afferent nerve signaling. Neurogastroenterol Motil 27: 53.

2. Blackshaw LA, Gebhart GF (2002) The pharmacology of gastrointestinal nociceptive pathways. Curr Opin Pharmacol 2: 642-649.

3. Booth CE, Shaw J, Hicks GA, Kirkup AJ, Winchester W, Grundy D (2008) Influence of the pattern of jejunal distension on mesenteric afferent sensitivity in the anaesthetized rat. Neurogastroenterol Motil 20: 149-158.

4. Keating C, Beyak M, Foley S, Singh G, Marsden C, Spiller R, et al. (2008) Afferent hypersensitivity in a mouse model of post-inflammatory gut dysfunction: role of altered serotonin metabolism. J Physiol 586: 4517-4530.

5. Deiteren A, Vermeulen W, Moreels TG, Pelckmans PA, De Man JG, De Winter BY (2014) The effect of chemically induced colitis, psychological stress and their combination on visceral pain in female Wistar rats. Stress 17: 431-444.

6. Ruyssers NE, De Winter BY, De Man JG, Ruyssers ND, Van Gils AJ, Loukas A, et al. (2010) Schistosoma mansoni proteins attenuate gastrointestinal motility disturbances during experimental colitis in mice. World J Gastroenterol 16: 703-712.

7. te Velde AA, Verstege MI, Hommes DW (2006) Critical appraisal of the current practice in murine TNBS-induced colitis. Inflamm Bowel Dis 12: 995-999.

8. Larsson M, Arvidsson S, Ekman C, Bayati A (2003) A model for chronic quantitative studies of colorectal sensitivity using balloon distension in conscious mice -- effects of opioid receptor agonists. Neurogastroenterol Motil 15: 371-381.

9. Kyloh M, Nicholas S, Zagorodnyuk VP, Brookes SJ, Spencer NJ (2011) Identification of the visceral pain pathway activated by noxious colorectal distension in mice. Front Neurosci 5: 16.

10. Ness TJ, Gebhart GF (1988) Colorectal distension as a noxious visceral stimulus: physiologic and pharmacologic characterization of pseudaffective reflexes in the rat. Brain Res 450: 153-169.

11. Mueller MH, Glatzle J, Kampitoglou D, Kasparek MS, Grundy D, Kreis ME (2008) Differential sensitization of afferent neuronal pathways during postoperative ileus in the mouse jejunum. Ann Surg 247: 791-802.

12. Wyndaele M, De Wachter S, De Man J, Minagawa T, Wyndaele JJ, Pelckmans PA, et al. (2013) Mechanisms of pelvic organ crosstalk: 1 . Peripheral modulation of bladder inhibition by colorectal distention in rats. J Urol 190: 765-771. 
13. Brusberg M, Ravnefjord A, Martinsson R, Larsson H, Martinez V, Lindstrom E (2009) The GABA(B) receptor agonist, baclofen, and the positive allosteric modulator, CGP7930, inhibit visceral painrelated responses to colorectal distension in rats. Neuropharmacology 56: 362-367.

14. Lindstrom E, Brusberg M, Ravnefjord A, Kakol-Palm D, Pahlman I, Noven A, et al. (2011) Oral baclofen reduces visceral pain-related pseudo-affective responses to colorectal distension in rats: relation between plasma exposure and efficacy. Scand J Gastroenterol 46: 652-662. 